\title{
Utilisation de la technologie pour améliorer les soins de santé dans les collectivités des Premières nations de la Saskatchewan
}

\author{
I Khan ${ }^{1 *}$, N Ndubuka ${ }^{2}$, K Stewart ${ }^{3,4}$, V McKinney $^{5}$ et I Mendez ${ }^{6}$
}

\section{Résumé}

Contexte : La Saskatchewan est une province qui compte plus d'un million d'habitants, dont plus de $13 \%$ sont des Autochtones qui vivent en grand nombre sur des terres de réserve. Malgré des efforts continus, l'accès aux soins de santé demeure un défi important pour ces autochtones, en particulier celles qui résident dans le Nord.

Objectif : Pour résoudre ce problème, les fournisseurs de soins de santé de la Saskatchewan intègrent de plus en plus l'usage de la technologie dans la prestation de divers services de santé. Cet article décrit diverses façons dont la technologie est utilisée dans les collectivités des Premières nations de la Saskatchewan.

Méthodologie : Au cours des dix dernières années, plusieurs projets pilotes ont été mis en œuvre à l'échelon communautaire et en milieu urbain, en collaboration avec des chefs des Premières nations et des fournisseurs de soins de santé. L'information recueillie sur ces projets pilotes a été complétée par une revue de la littérature et par des consultations auprès des collègues de la Northern Inter-Tribal Health Authority, de la Direction générale de la santé des Premières nations et des Inuits, de Santé Canada et de médecins de premier plan offrant des services dans les collectivités du Nord.

Résultats : De nombreuses technologies s'annoncent prometteuses pour faciliter la prestation en temps opportun de soins de santé de haute qualité. À titre d'exemple, la technologie robotique de téléprésence est une forme de télémédecine qui donne l'impression que le médecin est au chevet du patient, permettant ainsi d'offrir à distance des services cliniques en temps réel. L'amélioration de l'accès aux services Internet et la distribution de tablettes électroniques aux infirmières en santé communautaire ont amélioré l'accès des patients aux soins cliniques ainsi qu'à de l'information vitale sur les soins de santé. L'échographie robotisée est utilisée pour la prestation sur place de soins prénatals. La distribution de téléphones cellulaires aux patients séropositifs pour le VIH a amélioré l'observance du traitement antirétroviral et amélioré les résultats cliniques. Le Xpert MTB/RIF (complexe Mycobacterium tuberculosis) résistance à la rifampicine) est un test automatisé qui, par l'analyse d'échantillons d'expectorations non traités, peut détecter la présence de $M$. tuberculosis plus rapidement et avec une plus grande sensibilité et spécificité que le frottis classique basé sur la recherche de bacilles acido-alcoolo-résistants. De même, du matériel de communication à distance est utilisé dans le cadre des services de télémédecine pour la prestation de soins aux patients dans la collectivité. Panorama est un système exhaustif intégré de gestion de l'information en santé publique, qui a été conçu pour les professionnels de la santé publique et qui est actuellement mis en place dans 21 collectivités de la Saskatchewan.

Conclusion : Non seulement ces technologies novatrices semblent améliorer l'accès en temps opportun aux soins de santé et la qualité des soins dans les collectivités éloignées, mais elles contribuent également au bien-être des patients en évitant les transports inutiles et en réduisant au minimum les absences du travail et le temps passé loin de la famille. Bien que ces technologies n'offrent pas une solution pour certains déterminants de la santé pouvant influer sur l'incidence et la gravité des maladies infectieuses chez les Premières nations, elles semblent atténuer certains défis géographiques qui nuisent à la prestation des services de santé dans les communautés éloignées.

\author{
Affiliations \\ 1 Direction générale de la santé \\ des Premières nations et des \\ Inuits, Santé Canada, Région \\ de la Saskatchewan, Regina \\ (Saskatchewan) \\ ${ }^{2}$ Northern Inter-Tribal Health \\ Authority, Prince Albert \\ (Saskatchewan) \\ ${ }^{3}$ Saskatchewan Infectious \\ Disease Care Network, Saskatoon \\ (Saskatchewan) \\ ${ }^{4}$ Prévention et contrôle de la \\ tuberculose de la Saskatchewan, \\ Autorité régionale de santé \\ de Saskatoon, Saskatoon \\ (Saskatchewan) \\ ${ }^{5}$ Northern Medical Services, \\ Saskatoon (Saskatchewan) \\ ${ }^{6}$ Département de chirurgie, Royal \\ University Hospital, Saskatoon \\ (Saskatchewan)
}

Correspondance : ibrahim.khan@ hc-sc.gc.ca
Citation proposée : Khan I, Ndubuka N, Stewart K, McKinney V et Mendez I. Utilisation de la technologie pour améliorer les soins de santé dans les collectivités des Premières nations de la Saskatchewan. Relevé des maladies transmissibles au Canada. 2017;43(6):134-9. https://doi.org/10.14745/ccdr.v43i06a01f

\section{Introduction}

La Saskatchewan est une grande province, dont la taille équivaut à peu près à celle de la France. Plus de $13 \%$ du quelque million d'habitants de cette province sont des membres des Premières nations (Autochtones) dont plus de la moitié vit sur des terres de réserve (1-3). D'après les données de 2015, les taux de prévalence du virus de l'immunodéficience humaine $(\mathrm{VIH})$ sont de 47 cas pour 100000 membres des Premières nations vivant dans des réserves du centre-sud de la Saskatchewan et de 37 cas pour 100000 parmi ceux vivant dans des réserves du Nord (4). Les taux de tuberculose, de co-infection VIH-hépatite $\mathrm{C}$, de maladies chroniques (comme le diabète) et de lésions traumatiques, ainsi que les tendances en matière de toxicomanies chez les membres des Premières nations de la Saskatchewan, demeurent également les plus élevés au Canada 
$(2,4,5)$. Malgré des efforts continus, I'accès aux soins primaires et aux soins spécialisés demeure un défi important pour les membres des Premières nations, en particulier pour ceux qui vivent dans le Nord. L'accès en temps opportun à des services de diagnostic et aux procédures pose notamment problème depuis longtemps dans les collectivités éloignées et isolées $(2,3,5)$. Et ce sont les segments les plus vulnérables de la population, notamment les enfants, les femmes enceintes et les personnes âgées $(2,3,5)$, qui sont les plus touchés par cette difficulté d'accès aux services de santé.

Santé Canada, les Premières nations du Nord, les médecins des régions concernées et les autorités sanitaires de l'ensemble de la province explorent divers moyens novateurs pour offrir des services de santé dans les régions éloignées du Nord $(5,6,9)$. Ces efforts ont favorisé le développement d'une pensée innovatrice et le recours à diverses technologies, notamment des instruments diagnostiques numériques et portatifs, pour la prestation des services de santé dans ces collectivités (4-6). Le présent aperçu vise à décrire la façon dont la technologie a été appliquée aux services de santé dans les collectivités des Premières nations du nord de la Saskatchewan.

\section{Méthodologie}

Au cours des dix dernières années, plusieurs projets pilotes menés avec succès dans les collectivités du nord de la Saskatchewan ont ouvert la voie à une transformation de la prestation de services de santé $(5-7,11)$. Ces projets pilotes visaient notamment à créer des partenariats avec des chefs des Premières nations et des fournisseurs de soins de santé, à l'échelon communautaire et en milieu urbain, en vue d'établir des liens entre les services dans les réserves et ceux offerts hors réserve (6). Tous les auteurs participent à un certain nombre de ces initiatives. L'information recueillie sur ces projets pilotes a été complétée par une revue de la littérature et par des consultations auprès des collègues de la Northern Inter-Tribal Health Authority, de la Direction générale de la santé des Premières nations et des Inuits, de Santé Canada et des médecins de premier plan offrant des services dans les collectivités du Nord $(5,6,9,11-13,15)$.

L'Organisation mondiale de la Santé définit les technologies de la santé comme étant " l'application de connaissances et de compétences organisées sous la forme de dispositifs, de médicaments, de vaccins, de procédures et de systèmes mis au point pour résoudre un problème de santé et améliorer la qualité de la vie » (8). La technologie est utilisée dans le nord de la Saskatchewan pour améliorer l'accès des patients aux services de soins de santé et l'accès des professionnels de la santé aux ressources en ligne, pour évaluer la possibilité d'interventions diagnostiques à distance ainsi que pour améliorer la prise en charge d'affections précises dans les collectivités éloignées des Premières nations $(5,6)$. Aux fins du présent aperçu, nous nous intéressons principalement aux technologies numériques et aux outils portatifs de télédiagnostic.

\section{Résultats}

\section{Amélioration de l'accès des patients aux services de santé}

La technologie robotique de téléprésence est l'une des technologies visant à améliorer l'accès des patients aux soins de santé; cette technologie consiste en l'utilisation de robots dirigeables ("Rosie ») et d'appareils portatifs ("Doc in the Box») qui permettent aux patients et aux fournisseurs de soins de santé d'être face à face, malgré la distance qui les sépare. Cette technologie, qui a été mise au point en Californie, utilise un signal téléphonique Wi-Fi ordinaire pour établir une liaison vidéo bidirectionnelle $(5,6,9,11-13,15)$.

\section{Amélioration de l'accès des professionnels de la santé aux ressources en ligne}

Le Programme d'infostructure de la cybersanté de la Direction générale de la santé des Premières nations et des Inuits (région de la Saskatchewan) apporte un soutien aux collectivités des Premières nations qui vivent sur des réserves ainsi qu'à d'autres organismes de la province, afin d'améliorer la connectivité pour la mise en réseau des services. Aujourd'hui, la majorité des collectivités des Premières nations vivant dans des réserves en Saskatchewan ont accès à des services Internet. Des tablettes électroniques ont été fournies à un groupe d'infirmières en santé communautaire de la Saskatchewan, pour améliorer leur capacité d'accéder à des outils en ligne d'information sur les soins cliniques et les soins infirmiers en santé publique et à des outils d'éducation pour les patients visés par les interventions communautaires. Selon les résultats préliminaires, ce projet pilote a permis aux infirmières d'accéder rapidement à différentes ressources à l'aide de ces tablettes, durant la prestation de soins ambulatoires ou d'interventions communautaires dans les collectivités $(5,7,11)$.

\section{Évaluer le potentiel des technologies de télédiagnostic}

L'accès aux services diagnostiques, en particulier à l'échographie, fait grandement défaut dans les collectivités des Premières nations. L'éloignement et les longs temps de déplacement posent de grandes difficultés à cet égard. Dans le cadre d'une étude pilote réalisée en 2014, le Dr Mendez a utilisé la technologie robotique de téléprésence pour évaluer et traiter des enfants gravement malades vivant à Pelican Narrows dans le centre de la Saskatchewan $(6,15)$. Les résultats ont démontré que cette technologie était sécuritaire, cliniquement efficace et rentable, $63 \%$ des enfants gravement malades ayant pu être traités dans leur collectivité. Au cours des dix dernières années, le $D^{r}$ Mendez s'est fait le champion de l'application de cette technologie à distance et il a mené avec succès plusieurs projets pilotes, non seulement dans des collectivités du nord de la Saskatchewan, mais aussi dans le monde entier $(5,6,9,11,13)$. L'utilisation de la robotique de téléprésence et d'autres technologies a ouvert la voie à la transformation de 
la prestation des services de santé. Aujourd'hui, 11 robots médicaux et appareils portatifs sont utilisés en pratique clinique en Saskatchewan, soit plus que partout ailleurs au Canada (9).

Des spécialistes des maladies infectieuses de la Saskatchewan utilisent un appareil FibroScan portable pour la tenue de cliniques du foie dans les réserves (7). Le Dr Mendez et bon nombre d'autres fournisseurs de soins de santé se sont associés à des chefs des Premières nations et à des fournisseurs de soins de santé, à l'échelon communautaire et en milieu urbain, pour combler les lacunes et établir des liens entre les services de santé offerts aux Premières nations vivant dans des réserves et hors réserve $(5,6,9,11,12)$.

Le taux de nouveaux cas de tuberculose est très élevé dans les collectivités du Nord $(2,7)$. En plus du dépistage systématique de la tuberculose, une nouvelle technologie diagnostique du nom de Xpert-MTBC/RIF (complexe M. tuberculosis/résistance à la rifampicine) est actuellement mise à l'essai dans des collectivités du Nord, dans le cadre d'un projet pilote visant à offrir un test de dépistage rapide pour les patients que l'on croit atteints de tuberculose (10). Un résultat négatif à ce test permet d'écarter rapidement la tuberculose et de prévenir un isolement inutile. À l'inverse, un résultat positif favorise un diagnostic précoce et I'instauration rapide d'un traitement, réduisant ainsi l'exposition des autres membres de la collectivité à un cas de tuberculose évolutive $(17,18)$.

\section{Télésanté}

La télésanté est une technologie de vidéoconférence sur Internet qui est utilisée par bon nombre de fournisseurs de soins de santé pour évaluer des patients ou offrir des séances d'éducation et de formation, ainsi qu'à d'autres fins dans la prestation de divers services et programmes de santé. À l'heure actuelle, 39 collectivités des Premières nations de la Saskatchewan disposent d'équipement de télésanté $(7,16)$, et on s'attend à ce que le nombre de ces collectivités et la fréquence d'utilisation des services de télésanté augmentent au cours des prochaines années dans la province. Le tableau 1 résume un éventail d'objectifs en matière de soins de santé, les technologies de télésanté utilisées pour atteindre ces objectifs, leur application dans le domaine des soins de santé et les résultats préliminaires sur la santé.

Tableau 1 : Technologies utilisées pour améliorer les services de santé offerts aux membres des Premières nations de la Saskatchewan

\begin{tabular}{|l|l|l|l|}
\hline \multicolumn{1}{|c|}{ But } & Technologie & $\begin{array}{l}\text { Application } \\
\text { au domaine } \\
\text { des soins de } \\
\text { santé }\end{array}$ & $\begin{array}{l}\text { Exemples de } \\
\text { résultats }\end{array}$ \\
\hline $\begin{array}{l}\text { Améliorer } \\
\text { I'accès des } \\
\text { patients aux } \\
\text { soins de santé }\end{array}$ & $\begin{array}{l}\text { La technologie } \\
\text { robotique de } \\
\text { téléprésence } \\
\text { donne } \\
\text { I'impression } \\
\text { qu'un médecin se } \\
\text { trouve au chevet } \\
\text { du patient, alors } \\
\text { qu'il est en } \\
\text { réalité éloigné; } \\
\text { elle permet la } \\
\text { prestation }\end{array}$ & $\begin{array}{l}\text { Utilisée par des } \\
\text { médecins de } \\
\text { premier recours, } \\
\text { des infirmières, } \\
\text { des thérapeutes } \\
\text { et des travailleurs } \\
\text { des services } \\
\text { d'approche, } \\
\text { en médecine } \\
\text { d'urgence et } \\
\text { traumatologie, } \\
\text { ainsi que par des }\end{array}$ & $\begin{array}{l}\text { Accélérion } \\
\text { de la prise en } \\
\text { charge du VIH } \\
\text { et du triage de } \\
\text { troubles aigus, } \\
\text { amélioration } \\
\text { du bien-être et } \\
\text { de la sécurité } \\
\text { des patients, et } \\
\text { accès facile et } \\
\text { rapide aux }\end{array}$ \\
\hline
\end{tabular}

\begin{tabular}{|c|c|c|c|}
\hline But & Technologie & $\begin{array}{c}\text { Application } \\
\text { au domaine } \\
\text { des soins de } \\
\text { santé }\end{array}$ & $\begin{array}{l}\text { Exemples de } \\
\text { résultats }\end{array}$ \\
\hline $\begin{array}{l}\text { Améliorer } \\
\text { l'accès des } \\
\text { patients aux } \\
\text { soins de santé } \\
\text { (suite) }\end{array}$ & $\begin{array}{l}\text { à distance de } \\
\text { services cliniques } \\
\text { en temps réel. } \\
\text { Aussi appelée } \\
\text { «Doc in the } \\
\text { Box » }\end{array}$ & $\begin{array}{l}\text { professionnels de } \\
\text { la santé mentale, } \\
\text { des pédiatres et } \\
\text { des conseillers en } \\
\text { santé publique ou } \\
\text { sur le VIH }\end{array}$ & $\begin{array}{l}\text { services de } \\
\text { santé dans la } \\
\text { collectivité } \\
(5,6,9)\end{array}$ \\
\hline $\begin{array}{l}\text { Améliorer } \\
\text { l'accès aux } \\
\text { ressources } \\
\text { en ligne pour } \\
\text { les infirmières } \\
\text { en santé } \\
\text { communautaire } \\
\text { et leurs } \\
\text { gestionnaires }\end{array}$ & $\begin{array}{l}\text { Utilisation } \\
\text { de tablettes } \\
\text { électroniques } \\
\text { durant les } \\
\text { cliniques mobiles } \\
\text { de sensibilisation } \\
\text { et les visites }\end{array}$ & $\begin{array}{l}\text { Soins infirmiers } \\
\text { communautaires, } \\
\text { soins primaires, } \\
\text { médecins } \\
\text { hygiénistes et } \\
\text { équipes mobiles } \\
\text { de sensibilisation } \\
\text { dans les } \\
\text { collectivités }\end{array}$ & $\begin{array}{l}\text { Outil facile à } \\
\text { transporter, qui } \\
\text { offre un accès } \\
\text { rapide à des } \\
\text { ressources en } \\
\text { ligne sur les } \\
\text { soins infirmiers/ } \\
\text { les soins } \\
\text { cliniques/la santé } \\
\text { publique et à } \\
\text { d'autres outils } \\
\text { pédagogiques } \\
\text { pertinents et } \\
\text { qui permet } \\
\text { d'améliorer la } \\
\text { qualité des soins } \\
\text { et de gagner du } \\
\text { temps (11) }\end{array}$ \\
\hline \multirow[t]{3}{*}{$\begin{array}{l}\text { Améliorer la } \\
\text { prise en charge } \\
\text { d'affections } \\
\text { précises }\end{array}$} & $\begin{array}{l}\text { Technologie de } \\
\text { vidéoconférence } \\
\text { sur Internet }\end{array}$ & $\begin{array}{l}\text { Utilisée par } \\
\text { bon nombre } \\
\text { d'intervenants, } \\
\text { notamment des } \\
\text { fournisseurs de } \\
\text { soins de santé, } \\
\text { pour évaluer les } \\
\text { patients, offrir } \\
\text { des séances } \\
\text { d'éducation et de } \\
\text { formation, ainsi } \\
\text { qu'à d'autres fins } \\
\text { dans le cadre } \\
\text { de services et } \\
\text { programmes de } \\
\text { santé }\end{array}$ & $\begin{array}{l}\text { Actuellement } \\
\text { utilisée dans } \\
39 \text { centres de } \\
\text { santé relevant de } \\
\text { la compétence } \\
\text { des Premières } \\
\text { nations. Facilite } \\
\text { l'évaluation } \\
\text { régulière des } \\
\text { patients atteints } \\
\text { de tuberculose, } \\
\text { améliore } \\
\text { l'observance } \\
\text { des traitements, } \\
\text { augmente la } \\
\text { fréquence du } \\
\text { suivi et améliore } \\
\text { les résultats } \\
\text { du traitement } \\
(7,10,11,16)\end{array}$ \\
\hline & $\begin{array}{l}\text { Téléphones } \\
\text { cellulaires offerts } \\
\text { aux patients } \\
\text { séropositifs pour } \\
\text { le VIH }\end{array}$ & $\begin{array}{l}\text { Programmes } \\
\text { communautaires } \\
\text { de lutte contre } \\
\text { le VIH et la } \\
\text { tuberculose }\end{array}$ & $\begin{array}{l}\text { Améliore la } \\
\text { participation } \\
\text { aux cliniques, } \\
\text { les résultats du } \\
\text { traitement et la } \\
\text { gestion des cas, } \\
\text { et assure une } \\
\text { communication } \\
\text { régulière avec } \\
\text { les patients dans } \\
\text { le cadre de leur } \\
\text { suivi }(9,11)\end{array}$ \\
\hline & $\begin{array}{l}\text { Panorama est } \\
\text { un système } \\
\text { exhaustif intégré } \\
\text { de gestion de } \\
\text { l'information en } \\
\text { santé publique, } \\
\text { conçu pour les } \\
\text { professionnels en } \\
\text { santé publique }\end{array}$ & $\begin{array}{l}\text { Ce système } \\
\text { accélère } \\
\text { l'établissement } \\
\text { des stocks de } \\
\text { vaccins et la } \\
\text { surveillance dans } \\
\text { les collectivités } \\
\text { dans le cadre des } \\
\text { programmes }\end{array}$ & $\begin{array}{l}\text { Le système } \\
\text { aide les } \\
\text { professionnels à } \\
\text { collaborer plus } \\
\text { efficacement } \\
\text { pour gérer les } \\
\text { programmes de } \\
\text { vaccination, les } \\
\text { enquêtes sur }\end{array}$ \\
\hline
\end{tabular}




\section{Discussion}

\begin{tabular}{|c|c|c|c|}
\hline But & Technologie & $\begin{array}{c}\text { Application } \\
\text { au domaine } \\
\text { des soins de } \\
\text { santé }\end{array}$ & $\begin{array}{l}\text { Exemples de } \\
\text { résultats }\end{array}$ \\
\hline $\begin{array}{l}\text { Améliorer la } \\
\text { prise en charge } \\
\text { d'affections } \\
\text { précises } \\
\text { (suite) }\end{array}$ & & $\begin{array}{l}\text { de vaccination. } \\
\text { Utilisé par les } \\
\text { infirmières } \\
\text { en santé } \\
\text { communautaire ou } \\
\text { leurs gestionnaires } \\
\text { et les médecins } \\
\text { hygiénistes }\end{array}$ & $\begin{array}{l}\text { les éclosions } \\
\text { et la santé } \\
\text { familiale dans } \\
21 \text { collectivités. } \\
\text { L'objectif est } \\
\text { d'accroître le } \\
\text { nombre futur } \\
\text { de collectivités } \\
\text { participantes } \\
(7,10)\end{array}$ \\
\hline \multirow[t]{4}{*}{$\begin{array}{l}\text { Améliorer les } \\
\text { services de } \\
\text { diagnostic }\end{array}$} & $\begin{array}{l}\text { Échographie } \\
\text { robotisée } \\
\text { pour les soins } \\
\text { prénatals }\end{array}$ & $\begin{array}{l}\text { Fournisseurs de } \\
\text { soins prénatals }\end{array}$ & $\begin{array}{l}\text { Prestation plus } \\
\text { pratique des } \\
\text { soins, économies } \\
\text { de temps et } \\
d^{\prime} \text { argent et } \\
\text { déplacements } \\
\text { moins fréquents } \\
\text { pour les } \\
\text { patients, et } \\
\text { réduction des } \\
\text { répercussions } \\
\text { sur les familles }\end{array}$ \\
\hline & $\begin{array}{l}\text { Technologie } \\
\text { robotique de } \\
\text { téléprésence } \\
\text { pour le } \\
\text { diagnostic et le } \\
\text { traitement aux } \\
\text { points de service }\end{array}$ & $\begin{array}{l}\text { Fournisseurs de } \\
\text { soins primaires } \\
\text { spécialistes du } \\
\text { domaine }\end{array}$ & $\begin{array}{l}\text { Prestation plus } \\
\text { pratique des } \\
\text { soins, économies } \\
\text { de temps et } \\
\text { d'argent et } \\
\text { déplacements } \\
\text { moins fréquents } \\
\text { pour les } \\
\text { patients, et } \\
\text { réduction des } \\
\text { répercussions } \\
\text { sur les familles } \\
(5,6,9,11-13,15)\end{array}$ \\
\hline & $\begin{array}{l}\text { Appareil } \\
\text { FibroScan } \\
\text { portable }\end{array}$ & $\begin{array}{l}\text { Infirmières } \\
\text { spécialisées en } \\
\text { santé du foie } \\
\text { et médecins } \\
\text { spécialistes } \\
\text { des maladies } \\
\text { infectieuses }\end{array}$ & $\begin{array}{l}\text { Appareil utilisé } \\
\text { pour l'évaluation } \\
\text { et la stadification } \\
\text { de la fibrose } \\
\text { hépatique chez } \\
\text { les patients } \\
\text { atteints } \\
\text { d'hépatite B ou } \\
\text { C (7). Évaluation } \\
\text { faite dans la } \\
\text { collectivité }\end{array}$ \\
\hline & $\begin{array}{l}\text { Le Xpert MTB/ } \\
\text { RIF est un } \\
\text { nouveau test } \\
\text { moléculaire pour } \\
\text { le dépistage de } \\
\text { la tuberculose et } \\
\text { d'autres maladies }\end{array}$ & $\begin{array}{l}\text { Diagnostic rapide } \\
\text { de la tuberculose }\end{array}$ & $\begin{array}{l}\text { Outil de } \\
\text { diagnostic } \\
\text { rapide de la } \\
\text { tuberculose et } \\
\text { de prévention } \\
\text { précoce des } \\
\text { expositions } \\
\text { possibles, } \\
\text { récemment mis } \\
\text { en place dans } \\
\text { des collectivités } \\
\text { du Nord pour } \\
\text { appuyer la } \\
\text { stratégie de } \\
\text { lutte contre } \\
\text { la forte } \\
\text { incidence de } \\
\text { la tuberculose } \\
(10,17,18)\end{array}$ \\
\hline
\end{tabular}

Abréviations : VIH, virus de l'immunodéficience humaine; MTB/RI, Mycobacterium tuberculosis/ rifampicine
Le succès de l'utilisation de la robotique, d'Internet, des tablettes électroniques, des téléphones cellulaires dotés d'applications propres à certaines maladies, de la télésanté, de l'échographie à distance et de la scintigraphie hépatique, ainsi que des systèmes électroniques intégrés de gestion de l'information en santé publique et de tests de diagnostic rapide, montre que les technologies électroniques et numériques peuvent améliorer l'accès aux services de santé dans les collectivités éloignées des Premières nations. L'utilisation de la technologie a permis d'améliorer l'accès des professionnels de la santé à des renseignements à jour sur les soins de santé tout en offrant la possibilité d'améliorer la capacité de diagnostic dans les régions de la Saskatchewan qui en ont le plus besoin. Cette avancée a d'ores et déjà permis d'améliorer la rapidité des soins offerts aux clients et l'accès à un large éventail d'expertise médicale dans les collectivités éloignées du Nord. Ces technologies ont également accru l'instauration de traitements et de mesures prophylactiques comme la vaccination et amélioré l'accès à des services de première importance $(5,6,9,11,12,15,16)$. Le recours à la technologie dans les services de santé s'est en outre révélé rentable $(2,5,6,9,16)$.

L'utilisation accrue des technologies relatives aux soins de santé dans les collectivités éloignées du nord de la Saskatchewan a été bien accueillie par les patients. Ces technologies ont en effet permis d'accroître le bien-être des patients, en leur évitant des déplacements inutiles et en leur permettant de rester avec leur famille, dans leur collectivité. Ces technologies ont également favorisé une prise en charge individuelle de la santé.

Bien que ces technologies aient contribué à combler certaines lacunes dans la prestation des soins primaires dans les collectivités des Premières nations, elles comportent aussi certaines limites qui doivent être prises en compte : I'utilisation de la technologie ne peut jamais remplacer complètement les avantages d'une rencontre en personne entre un patient et un fournisseur de soins de santé (14); la technologie peut exiger beaucoup de ressources et elle nécessite une formation $(11,16)$. Il faudra du temps et des efforts pour intégrer l'utilisation de la technologie, et cette intégration nécessitera de vastes consultations auprès des collectivités des Premières nations, des fournisseurs locaux de soins de santé et des dirigeants des Premières nations, ainsi que des représentants de différents ordres de gouvernement $(5,6,11,16)$. Enfin, même si les technologies n'offrent pas une solution pour certains déterminants de la santé qui peuvent influer sur l'incidence et la gravité des maladies infectieuses dans les collectivités des Premières nations, leur utilisation semble néanmoins atténuer certaines difficultés géographiques qui nuisent à la prestation des services de santé dans les collectivités éloignées.

Comme les résultats présentés ici ne sont que préliminaires, il serait très utile de faire une évaluation systématique officielle des pratiques actuelles, en vue d'un élargissement futur de I'utilisation de ces technologies. II serait également utile d'examiner comment ces pratiques et les leçons apprises des projets pilotes pourraient être appliquées dans d'autres régions pour combler les lacunes dans les services et améliorer les résultats sur la santé à long terme. 


\section{Conclusion}

En Saskatchewan, un nombre croissant de médecins et d'autres professionnels de la santé ont aujourd'hui recours à des technologies novatrices pour améliorer l'accès aux services de santé. Bien que des lacunes persistent et que d'autres recherches devront être menées, l'utilisation de ces technologies semble offrir un moyen sûr, efficace et rentable pour améliorer les soins de santé dans les collectivités éloignées des Premières nations et d'autres collectivités.

\section{Déclaration des auteurs}

I. K. - conceptualisation, recherche, rédaction de l'ébauche initiale, méthodologie, logiciel, validation, examen et révision; K. S., N. N. et V. M. - partage des connaissances, examen et révision

\section{Conflit d'intérêt}

I. K., N. N., V. M. et I. M. n'ont aucun conflit d'intérêts à déclarer.

K. S. est consultant et conseiller auprès de Gilead Sciences, AbbVie et Merck et a reçu des subventions de Gilead, AbbVie, Merck, Roche, Vertex Pharmaceutiques et ViiV Soins de santé, mais n'a aucun intérêt dans quelque société pharmaceutique ni entreprise de soins de santé.

\section{Remerciements}

Nous tenons à remercier sincèrement $D^{r e}$ Katherine Hennessy et Dr Normen Ducharme de Santé Canada pour leur soutien, leur expertise et leur contribution à la préparation du présent article, ainsi que pour leur excellent travail dans ce domaine. Nous leur sommes profondément reconnaissants de leur engagement soutenu envers la santé des collectivités du Nord.

\section{Financement}

Aucun.

\section{Références}

1. Bureau of Statistics, Government of Saskatchewan. Disponible à l'adresse: http://www.stats.gov.sk.ca/.

2. Santé Canada. Rapport annuel sur l'état de santé des membres des Premières Nations vivant dans la province de la Saskatchewan 2012 [Internet]. Disponible à l'adresse : http://publications.gc.ca/site/eng/search/search.html?st=1\& $\mathrm{ssti}=1$ \&ast $=$ First + Nations + Health + Status + Report $+2012 \& \mathrm{cn}$ $\mathrm{st}=\& \_e=0 n \& \_f=0 n \& \_a d o f=0 n$.

3. Statistique Canada. Janine C. Coup d'oeil sur la santé, Difficulté liée à l'accès aux services de soins de santé au Canada [Internet], le 8 décembre 2016. Disponible à l'adresse : http://www.statcan.gc.ca/pub/82-624-x/2016001/ article/14683-fra.htm.

4. Charlton J. Saskatoon StarPhoenix, Sask First Nations could turn corner on HIV by 2020 [Internet]. Disponible à l'adresse : http://thestarphoenix.com/health/sexual-health/sask-firstnations-could-turn-corner-on-hiv-by-2020.

5. Dr. Mendez. CTV News Saskatchewan interview. 'Doctor in a box' soon to be used in clinical trails; November 23, 2013. Disponible à l'adresse : http://saskatoon.ctvnews.ca/doctorin-a-box-soon-to-be-used-in-clinical-trials-1.1557674.

6. Government of Saskatchewan [Internet]. Pioneering Robotics Technology Benefits Patients in the North, Sept 2016.

Disponible à l'adresse : https://www.saskatchewan.ca/ government/news-and-media/2016/september/27/robot.

7. Khan I, First Nations Inuit Health-Health Branch, Health Canada eHealth program communication 2017, Saskatchewan Region.

8. World Health Organization. Health Technology Assessment [Internet]. Disponible à l'adresse : http://www.who.int/ medical_devices/assessment/en/.

9. Ellen B. Five ways robots are delivering health care in Saskatchewan [Internet]. CBC News Saskatchewan, Feb 2015. Disponible à l'adresse : http://www.cbc.ca/news/ canada/saskatchewan/5-ways-robots-are-delivering-healthcare-in-saskatchewan-1.2966190.

10. Khan I, Ndubuka N. 2017 TB program communication. Health Canada. 2017.

11. Mendez I, Van den Hof M. Mobile remote-presence devices for point-of-care health care delivery. CMAJ. 2013;185(17):1512-6. DOI: http://dx.doi.org/10.1503/ cmaj.120223. PubMed (https://www.ncbi.nlm.nih.gov/ pubmed/23775612?dopt=Abstract).

12. Mendez I, Jong M, Keays-White D, Turner G. The use of remote presence for health care delivery in a northern Inuit community: a feasibility study. International Journal of Circumpolar Health. 2013;72(1):10. DOI: http://dx.doi. org/10.3402/ijch.v72i0.21112. PubMed (https://www.ncbi. nlm.nih.gov/pubmed/23984292?dopt=Abstract).

13. Lovo Grona S, Bath B, Bustamante L, Mendez I. Use of a remote presence robot to improve access to physical therapy for people with chronic back disorders in an underserved community. Physiotherapy Canada. 2017;69(1):14-9. DOI: http://dx.doi.org/10.3138/ptc.2015-77. PubMed (https:// www.ncbi.nlm.nih.gov/pubmed/28154440?dopt=Abstract).

14. Buckeridge D. Session de plénière de la conférence de Santé publique 2016 du L'Association canadienne de santé publique [Internet]. 2016. Disponible à l'adresse : http:// www.cpha.ca/fr/conferences/archives/conf2016/plenaries. aspx.

15. Adams S, Burbridge B, Badea, A, Langford L, Vergara $V$, Bryce R, Bustamante L, Mendez I, Babyn P. Initial Experience Using a Telerobotic Ultrasound System to Perform Adult Abdominal Examinations. Canadian Association of Radiologists Journal. 2016. DOI: http://dx.doi.org/10.1016/j. carj.2016.08.002.

16. Abdullah $\mathrm{N}$, Nancy C. Telehealth in rural Canada, medicine and technology [Internet]. UWOMJ 2014;83(1):49-50. 
Disponible à l'adresse : http://www.uwomj.com/wp-content/ uploads/2014/10/v82no1_16.pdf.

17. Luetkemeyer AF, Firnhaber $C$, Kendall MA et al. Evaluation of Xpert MTB/RIF Versus AFB Smear and Culture to Identify Pulmonary Tuberculosis in Patients with Suspected Tuberculosis From Low and Higher Prevalence Settings. Clin Infect Dis. 2016;62(9):1081-8. DOI: http://dx.doi. org/10.1093/cid/ciw035. PubMed (https://www.ncbi.nlm.nih. gov/pubmed/26839383?dopt=Abstract).
18. Oxlade O, Sugarman J, Alvarez GG. Xpert $®$ MTB/RIF for the Diagnosis of Tuberculosis in a Remote Arctic Setting: Impact on Cost and Time to Treatment Initiation. PLoS One. 2016 Mar 18;11(3):e0150119. DOl: http://dx.doi.org/10.1371/ journal.pone.0150119. PubMed (https://www.ncbi.nlm.nih. gov/pubmed/26990299?dopt=Abstract).

\section{Lignes directrices canadiennes sur les} infections transmissibles sexuellement

\section{Mise à jour de l'application mobile (mai 2017)}
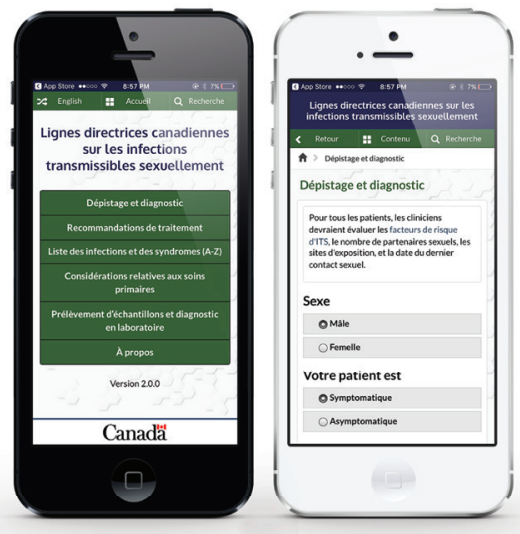

】+】

Agence de la santé Public Health publique du Canada Agency of Canada

\section{Nouveautés de la version 2.0.0}

- Mise à jour du contenu

- Amélioration de l'interactivité

- Corrections de bogues et amélioration des performances

\section{Téléchargez-la aujourd'hui}

Téléchargement gratuit

\section{Recherchez:}

"Lignes directrices CDN ITS "

Canadä 